\title{
Prognostic Value of Electrocardiographic Abnormalities and Troponin-I Elevation in Hospitalized COVID-19 Patients
}

\author{
Anisul Awal ${ }^{1}$, Kazi Shamim Al Mamun², Mohammed Rezaul Karim ${ }^{3}$, Md Saif Uddin Azad ${ }^{4}$, Farid Uddin Ahmed ${ }^{5}$
}

\begin{abstract}
:
Background: Corona Virus Disease (COVID -19) patients present mainly with respiratory manifestations and viral pneumonia. The cardiovascular presentation includes early signs of acute myocardial injury. Troponin elevation is a frequent laboratory finding in hospitalized patients with the disease, and may reflect direct vascular injury or nonspecific supply-demand imbalance. In this work, we assessed the correlation between different ranges of Troponin elevation, Electrocardiographic (ECG) abnormalities and mortality.
\end{abstract}

Methods and materials: It was a prospective observational study, conducted in four tertiary care Private Hospitals of Chattogram City of Bangladesh. The study enrolled 181 consecutive patients admitted to hospital from June 01, 2020 to December 31, 2020 due to Covid-19 disease on the basis of presentation of signs and symptoms severity. Upon admission, routine investigations cTnl and ECG were carried out.

Results: Mean age of the patients was $54.3 \pm 7.3$ years with $63.5 \%$ male. Hypertension was the most common comorbidity followed by diabetes and obesity. $57.1 \%$ of the patients had abnormal ECG. Abnormal axis deviation [26\% (left axis deviation $23.9 \%$ vs right axis deviation
$2.0 \%)$ ], Poor R wave progression $(22.9 \%)$, $T$ inversion $(14.5 \%)$, left ventricular hypertrophy (LVH) $(11.4 \%)$ followed by ST segment depression (8.3\%) were major findings observed in the study population. Presence of LVH ( $p=0.008)$, ST segment elevation ( $p \leq 0.001)$, ST segment depression $(p \leq 0.001)$ and $T$ inversion $(p=0.003)$ showed statistically significant association with Severe COVID-19 disease. $48.2 \%$ had raised cTnl level. Thirteen $\mathbf{( 7 . 2 \% )}$ patients expired in hospital. The mortality rate increased with incrementally higher troponin group: $12 / 18$ than mildly elevated troponin $1 / 63(p<0.01)$. The presence of an abnormal ECG finding resulted in significant in the intermediate Troponin elevation group $(0.05-1 \mathrm{ng} / \mathrm{ml})$ but not in the low $(<0.05 \mathrm{ng} / \mathrm{ml})$ or high ( $>$ $1 \mathrm{ng} / \mathrm{ml}$ ) Troponin elevation groups. There were statistically significant association between between cTnl level and death; and between ECG findings and death.

Conclusion: Study conclude that Troponin-I level and ECG are a prognostic factor for mortality in hospitalized COVID-19 patients.

Key words: COVID-19, Cardiac troponin I, Electrocardiography; Mortality.

(Bangladesh Heart Journal 2021; 36(2): 105-112)

Introduction:

In December 2019, a cluster of unusual cases of pneumonia were reported from Wuhan, China. Later on, the causative agent was identified as severe acute respiratory syndrome coronavirus 2 (SARS CoV2) which

1. Assistant Professor, Department of Cardiology, Chattogram Medical College Hospital.

2. Consultant, Department of Cardiology, $\mathrm{CMCH}$

3. Consultant, Department of Medicine, Park View Hospital, Chattogram

4. Consultant, Department of Cardiology, Ma-O Shishu Hospital, Chattogram

5. Assistant Professor; Department of Community Medicine, Rangamati Medical College

Address of Correspondence: Dr. Anisul Awal, MD, FCPS, Assistant Professor, Department of Cardiology, CMCH, Cell: 01977266943, Email: anisul.awal@yahoo.com

DOI: https://doi.org/10.3329/bhj.v36i2.56036

Copyright $\odot 2017$ Bangladesh Cardiac Society. Published by Bangladesh Cardiac Society. This is an Open Access articles published under the Creative Commons Attribution-NonCommercial 4.0 International License (CCBY-NC). This license permits use, distribution and reproduction in any medium, provided the original work is properly cited and is not used for commercial purposes. 
is a highly infectious single stranded RNA virus. This novel viral infection was subsequently termed as coronavirus disease 2019 (COVID 19). ${ }^{1}$ Soon COVID19 had spread rapidly to most of the countries in the world. Consequently, on 11th March 2020, the world health organization (WHO) declared COVID-19 as "pandemic", an emergency of international concern, requiring urgent public health interventions to control the disease.

SARS CoV2 binds to Angiotensin converting enzyme (ACE2) receptors with help of its spike protein and thus gains access into the human cell. ${ }^{2}$ ACE 2 receptors are expressed on pneumocytes (both type- 1 and type-2) and endothelial cells. Though the virus mainly targets the respiratory system, other organ involvement is also common.1-3 Research demonstrates that various tissues, including the myocardium of the heart, express ACE2 protein on their cellular surface. ${ }^{3}$

The clinical presentation of COVID-19 is remarkably heterogeneous; although it was initially considered a respiratory illness, multiple organ systems are often affected. ${ }^{3}$ The heart is one of the most critical organs and it is particularly probable that COVID-19 also contributes to viral myocardial damage, which could also be considered one of the leading causes of COVID-19 patients' death. ${ }^{4}$ Myocardial injury, as heralded by elevated troponin levels, was previously observed in various medical conditions, including pneumonia, and was found to be correlated with adverse outcomes such as cardiac complications and death. 5,6 To date, several studies have described myocardial injury in patients diagnosed with COVID-19.7-11

Electrocardiographic (ECG) and Troponin I are two highly available commonly used parameters to detect myocardial injury and these can be used to predict the prognosis of COVID 19. ${ }^{12}$ This study was thus conducted to further evaluate the incidence of myocardial injury in COVID-19 hospitalized patients, to characterize risk factors for myocardial injury among these patients, to determine its prognostic significance by reviewing admission troponin and ECG findings in a group of patients from Bangladesh.

\section{Methods:}

Study subjects and design

It was a prospective observational study was conducted in four tertiary care Private Hospitals of Chattogram City of Bangladesh includes- CSCR Hospital, Delta Health Care, Ctg. Ltd., Park View Hospital Ltd. \& Ma-O- Shishu Hospital. The study enrolled 181 consecutive patients admitted to hospital from June 01, 2020 to December
31, 2020 due to Covid-19 disease on the basis of presentation of signs and symptoms severity. All patients were diagnosed and graded as per the National guideline. ${ }^{14}$ The diagnosis of COVID-19 was confirmed with real-time reverse transcriptase-polymerase chain reaction (RT-PCR). Clinical data was collected by the attending physician during hospitalization of patients within 48 hours. This study complies with the edicts of the 1975 Declaration of Helsinki, and approval was taken by the institutional ethics board of each hospitals. ${ }^{13}$ Informed consent was taken from the attendants and stable patients.

We considered major adverse event (MAE) the composite of all-cause death and respiratory failure requiring orotracheal intubation during the hospitalization. Written informed consent was waived by the designated hospital's ethics committee for patients with emerging infectious diseases.

The clinical data of patients were collected including demographics, clinical symptoms, and signs, co-existing conditions, imaging findings, laboratory results as per case record form. To detect myocardial injury 12-lead ECG and cardiac troponin I was done at admission.

\section{Electrocardiogram analysis}

All 12-lead electrocardiograms recorded on admission and review it later (paper speed of $25 \mathrm{~mm} / \mathrm{s}$ and 1 millivolt equivalent to $10 \mathrm{~mm}$ ) were analyzed off-line. According to pre-defined criteria, two expert cardiologists blinded to clinical information and non-invasive diagnostic results, independently evaluated the ECG.

The considered parameters were as follows: rhythm (sinus, supraventricular or ventricular arrhythmias); heart rate; PR and QRS durations; QT and QTcorrected (using Bazett or Fridericia formula when the heart rate was $>90$ beats per minute was used) intervals; conduction disturbances (atrioventricular block, bundle branch block, or fascicular block) and ST-T segment alterations. The ST-segment deviation was measured as the height difference (in millimeters) between the $\mathrm{J}$ point and the isoelectric line (TP segment).

T wave was analyzed in all 12 leads and classified as normal (positive in all leads apart from III, aVR, V1, with voltage $\geq 0.1 \mathrm{mV}$ ), inverted (negative in any lead except III, aVR, V1, with voltage $\geq 0.1 \mathrm{mV}$ ) or flat (voltage $<0.1 \mathrm{mV}$ ). Pathological $Q$ waves were identified as any $Q$ wave $>40$ $\mathrm{ms}$ wide, $>2 \mathrm{~mm}$ deep or $>25 \%$ of QRS complex depth.

According to the ESC guidelines (Ibanez et al., 2018; Roffi et al., 2016), ST-T segment alterations were 
classified as primary if suggesting acute ischemia. Left ventricular hypertrophy (LVH) was defined using Sokolow-Lyon criteria ( $\mathrm{S}$ in $\mathrm{V} 1+\mathrm{R}$ in $\mathrm{V} 5$ or $\mathrm{V} 6 \geq 35 \mathrm{~mm}$ or $\mathrm{R}$ in $\mathrm{aVL} \geq 11 \mathrm{~mm}$ ) with or without secondary ST-T segment changes.

Electrocardiogram was defined as abnormal for any patient if ischemia alterations, left ventricular hypertrophy, tachy or bradyarrhythmias, and any new atrioventricular $(\mathrm{AV})$, bundle branch blocks, or significant morphology alterations (e.g., new $Q$ pathological waves) were present. Otherwise, patients presenting sinus rhythm without previously described alterations were reported as normal.

\section{Troponin I:}

Cardiac troponin, along with inflammatory and thrombotic markers, may aid clinicians in determining stage classification and stratifying risk for myocardial injury among patients with coronavirus disease 2019 (COVID19), according to a review article published in the Journal of the American College of Cardiology.

Increases in cardiac troponin that are suggestive of myocardial injury are common in patients with COVID19 and are associated with arrhythmias and death. These increases more frequently occur in patients who have chronic cardiovascular conditions and in those who present with severe COVID-19.

Elevated troponin levels above the $99^{\text {th }}$ percentile can be classified as chronic myocardial injury, acute nonischemic myocardial injury, or acute myocardial infarction. Chronic myocardial injury "is likely the etiology for many [patients with] COVID-19 because of the high prevalence of chronic cardiovascular conditions," noted the researchers. "These elevations are true positives for myocardial injury and associated with an adverse prognosis even without intercurrent disease."

We collected cardiac biomarkers, troponin I (Tn I), creatinine kinase $(\mathrm{CK})$, and pro- brain natriuretic peptide (Pro-BNP). Troponin I levels were defined as elevated if they were above the "high-sensitive" assay specific upper reference limit (cut-off of $0.05 \mathrm{ng} / \mathrm{ml}$ ).

\section{Clinical data}

Demographic characteristics (age and sex), clinical data (symptoms, co-morbidities, laboratory findings \& imaging) and therapy were collected from electronic medical records. Severe COVID-19 was defined as meeting arterial oxygen saturation $\leq 93 \%$ at rest or $\mathrm{PaO} 2$ / $\mathrm{FiO} 2 \leq 300 \mathrm{~mm} \mathrm{Hg}$. We did not include respiratory rate $\geq 30$ breaths/min according to the Diagnosis and Treatment Plan of COVID-19 suggested by National
Health Commission of China due to the considerable inter-observer variability (Yang et al., 2020).

\section{Statistical analysis}

Categorical variables are presented as percentages, and continuous variables as mean and standard deviation (SD). Categorical variables are gender, symptoms such as chest pain, SOB, co-morbidities (Cardiovascular disease, Chronic Lung disease, smoking, obesity, Hypertension, diabetes) ECG pattern, during hospitalization. The Chi-Squared exact test for categorical variable and independent t-test for continuous variables were used. The differences in the means for Tnl, d-dimer, C-reactive protein $\mathrm{CRP}$, creatinine phosphokinase $\mathrm{CPK}$, creatinine kinase-MB CKMB, Serum Urea, age temperature, respiratory rate, body mass index BMI, number of co-morbidities were analyzed using independent t-test. Multivariate logistic regression analysis was used to assess the association between severity status (critical and non-critical). All statistical analyses will be performed with SPSS, version 24.0 with p> 0.05 as a level of significance.

\section{Results:}

The study included 181 patients with a mean age of $54.3 \pm 7.3$ years. Majority of them were male $(63.5 \%)$ and belonged to the age group of 31-50years [ $n=73(40.3 \%)$ ]. Patients who were referred to ICU in view of severe disease constituted about $10.4 \%(n=19)$ of the total cases. About $38.6 \%(n=70)$ of the cases had comorbidities. The predominant co-morbidity among patients who were discharged (non-severe COVID-19 disease) and those who got referred to ICU (Severe COVID-19 disease) was hypertension (58.0\% vs $89.4 \%$ ), followed by diabetes mellitus ( $56.1 \%$ vs $57.8 \%$ ). Cough $(67.9 \%)$ was the most common symptom at presentation, followed by fever (64.6\%) and Fatigue (57.4\%), among cases which were discharged from the hospital. However, patients with severe disease had breathlessness (19.3\%) as their principal presenting complaint. The demographic and clinical characteristics of the patients in the present study, by the outcome status are depicted in Table I.

Analysis of the characteristics of the patients' Electrocardiogram, done at/near the time of admission revealed abnormal ECG in $57.1 \%(n=96)$ of the patients focused on table-II.

Table II depicts that, significantly higher proportion of patients with abnormal ECG at admission expired inhospital compared to patients having normal ECG. 
Table-I

Demographic and clinical characteristics of patients $(n=181)$ admitted with COVID-19 by outcome.

\begin{tabular}{|c|c|c|c|c|}
\hline Variables & $\begin{array}{c}\text { Total }(\%) \\
\text { N-181 }\end{array}$ & $\begin{array}{c}\text { Discharged/ Non severe } \\
(n=162) \\
\text { Frequency }(\%)\end{array}$ & $\begin{array}{l}\text { ICU support/Severe } \\
(n=19) \\
\text { Frequency }(\%) \\
\end{array}$ & P-value \\
\hline \multicolumn{5}{|l|}{ Gender } \\
\hline Male & $115(63.5)$ & $102(62.9)$ & $13(68.4)$ & 0.001 \\
\hline Female & $66(36.4)$ & $60(37.0)$ & $06(31.5)$ & \\
\hline \multicolumn{5}{|l|}{ Age group (in years) } \\
\hline $18-30$ & $38(20.9)$ & $37(22.8)$ & $01(5.2)$ & $0.000 * *$ \\
\hline $31-50$ & $73(40.3)$ & $70(43.2)$ & $03(15.7)$ & \\
\hline $51-70$ & $64(35.3)$ & $53(32.7)$ & $11(57.8)$ & \\
\hline$>70$ & $6(3.3)$ & $2(1.2)$ & $4(21.0)$ & \\
\hline Age (Mean \pm SD) & $54.3 \pm 7.3$ & $53.4 \pm 8.9$ & $55.2 \pm 5.7$ & 0.001 \\
\hline \multicolumn{5}{|l|}{ Co-morbidities } \\
\hline Hypertension & $111(61.3)$ & $94(58.0)$ & $17(89.4)$ & 0.02 \\
\hline Diabetes Mellitus & $102(56.3)$ & $91(56.1)$ & $11(57.8)$ & 0.05 \\
\hline Smoking & $69(38.1)$ & $65(40.1)$ & $04(21.0)$ & 0.04 \\
\hline Hyperlipidaemia & $78(43.0)$ & $73(45.0)$ & $05(26.3)$ & 0.01 \\
\hline Chronic Kidney Disease & $13(7.1)$ & $09(5.5)$ & $04(21.0)$ & 0.05 \\
\hline Coronary Artery Disease & $15(8.2)$ & $12(7.4)$ & $03(15.7)$ & 0.06 \\
\hline \multicolumn{5}{|l|}{ Symptomatology } \\
\hline Fever & $117(64.6)$ & $109(67.2)$ & $08(42.1)$ & 0.793 \\
\hline Cough & $123(67.9)$ & $112(69.1)$ & $11(57.8)$ & 0.534 \\
\hline Breathlessness & 35 (19.3) & $30(18.5)$ & $05(26.3)$ & 0.067 \\
\hline Fatigue & $104(57.4)$ & $98(60.4)$ & $06(31.5)$ & 0.051 \\
\hline Chest pain & $54(29.8)$ & $47(29.0)$ & $07(36.8)$ & 0.063 \\
\hline
\end{tabular}

${ }^{*} p<0.05,{ }^{* *} p<0.01$ (statistically significant)

Table-II

Association between ECG change and outcome

\begin{tabular}{lccc}
\hline ECG change & Survived $(n=168) \%$ & Expire in hospital $(n=13) \%$ & $P$ value \\
\hline Normal & $72(42.8)$ & $1(7.7)$ & 0.003 \\
Abnormal & $96(57.1)$ & $12(92.3)$ & \\
\hline
\end{tabular}

Among abnormal ECG, abnormal axis deviation [26\% (left axis deviation $23.9 \%$ vs right axis deviation $2.0 \%$ )], Poor R wave progression (22.9\%), T inversion (14.5\%), left ventricular hypertrophy (LVH) $(11.4 \%)$ followed by ST segment depression $(8.3 \%)$ were major findings observed in the study population. Presence of LVH $(p=0.008)$, ST segment elevation $(p \leq 0.001)$, ST segment depression $(p \leq 0.001)$ and $T$ inversion $(p=0.003)$ showed statistically significant association with Severe COVID19 disease (Table III). Sinus Tachycardia was noted $55.2 \%$ (53) in abnormal ECG cases. (Table-III)

Patients are categorized according to guidelines- mild, moderate and severe cases. Maximum patients were mild illness $60.2 \%$ and $92.3 \%$ were death from severe cases $10.4 \%$ which were statistically significant $(p=0.001)$. Table-IV

The mortality rate increased with incrementally higher troponin group: 12 out of 18 and only 1out of 63 in mildly elevated troponin $(p<0.01)$. The presence of an abnormal ECG finding resulted in significant in the intermediate Troponin elevation group $(0.05-1 \mathrm{ng} / \mathrm{ml})$ but not in the low $(<0.05 \mathrm{ng} / \mathrm{ml})$ or high $(>1 \mathrm{ng} / \mathrm{ml})$ Troponin elevation groups.

Thirteen patients who were expired or death with raised troponin- I and this association was statistically significant (Table V). 
Table-III

Abnormal Electrocardiographic characteristics of patients admitted with COVID 19, overall and by outcome status $(n=181)$.

\begin{tabular}{lcccc}
\hline Variables & $\mathrm{N}=96(\%)$ & $\begin{array}{c}\text { Discharged/ } \\
\text { Non severe }(\mathrm{n}=69) \\
\text { Frequency }(\%)\end{array}$ & $\begin{array}{c}\text { ICU support/ } \\
\text { Severe }(\mathrm{n}=27) \\
\text { Frequency }(\%)\end{array}$ & $\begin{array}{c}\text { P-value } \\
\text { Atrial premature complex }\end{array}$ \\
Ventricular premature complex & $03(3.1)$ & $02(2.8)$ & $01(3.7)$ & 0.625 \\
AV block & $04(4.1)$ & $03(4.3)$ & $01(3.7)$ & 0.342 \\
RBBB & $01(1.0)$ & 0 & $01(3.7)$ & $0.004^{*}$ \\
LBBB / LAHB & $02(2.0)$ & $02(2.8)$ & $01(3.7)$ & 0.625 \\
Left ventricular hypertrophy & $02(2.0)$ & $01(1.4)$ & $03(11.1)$ & 0.068 \\
ST elevation & $11(11.4)$ & $8(11.5)$ & $04(14.8)$ & $0.008^{*}$ \\
ST depression & $04(4.1)$ & 0 & $06(22.2)$ & $0.001^{*}$ \\
T inversion & $08(8.3)$ & $02(2.8)$ & $04(14.8)$ & 0.001 \\
Poor R wave progression & $14(14.5)$ & $10(14.4)$ & $02(7.4)$ & 0.003 \\
Abnormal axis deviation & $22(22.9)$ & $20(28.9)$ & $04(14.8)$ & 0.756 \\
$\quad$ Left axis & $25(26.0)$ & $21(30.4)$ & $0.3)$ & 0.340 \\
$\quad$ Right axis & $23(23.9)$ & $19(27.1)$ & 0 & \\
\hline
\end{tabular}

${ }^{*} p<0.05,{ }^{* *} p<0.01$ (statistically significant), AV block-atrioventricular block, RBBB-right bundle branch block, LBBB-left bundle branch block, LAHB-left anterior hemiblock

Table-IV

Clinical severity and outcome

\begin{tabular}{lcccc}
\hline Variables & $\begin{array}{c}\text { Total } \\
(\mathrm{n}=181)\end{array}$ & $\begin{array}{c}\text { Discharged }(\mathrm{n}=168) \\
\text { Frequency }(\%)\end{array}$ & $\begin{array}{c}\text { Death }(\mathrm{n}=13) \\
\text { Frequency }(\%)\end{array}$ & $\begin{array}{c}\text { P-value } \\
\text { Clinical category }\end{array}$ \\
$\quad 109(60.2)$ & $109(64.8)$ & 0 & 0.001 \\
$\quad$ Mild & $53(29.2)$ & $52(30.9 \%)$ & $01(7.6)$ & $12(92.3)$ \\
$\quad$ Moderate & $19(10.4)$ & $07(4.1)$ & \\
Severe/critical & &
\end{tabular}

${ }^{*} p<0.05,{ }^{* *} p<0.01$ (statistically significant)

Table-V

Association between Troponin I, ECG and mortality.

\begin{tabular}{|c|c|c|c|c|}
\hline Variables & $\begin{array}{c}\text { Overall } \\
(n=181)\end{array}$ & $\begin{array}{c}\text { ECG changes } \\
(n=96)\end{array}$ & $\begin{array}{c}\text { No ECG changes } \\
(n=85)\end{array}$ & P-value \\
\hline \multicolumn{5}{|l|}{ Clinical category } \\
\hline Initial troponin $(\mathrm{ng} / \mathrm{mL})$ & $0.21 \pm 1.73$ & $0.8 \pm 4.06$ & $0.09 \pm 0.37$ & 0.17 \\
\hline Maximum troponin & $2.16 \pm 16.83$ & $3.50 \pm 12.63$ & $1.87 \pm 17.62$ & 0.01 \\
\hline Troponin group & & & & $<0.01$ \\
\hline$\leq 0.05$ & $100(55.2 \%)$ & $22(22.9 \%)$ & $78(91.7 \%)$ & \\
\hline $0.05-1.00$ & $63(41.9 \%)$ & $58(60.4 \%)$ & $05(5.8 \%)$ & \\
\hline$>1.00$ & $18(11.0 \%)$ & $16(16.6 \%)$ & $02(2.3 \%)$ & \\
\hline Mortality & $13(7.1 \%)$ & $12(12.5 \%)$ & $01(1.1 \%)$ & 0.09 \\
\hline
\end{tabular}

${ }^{*} p<0.05,{ }^{* *} p<0.01$ (statistically significant)

\section{Discussion:}

COVID-19 has been shown to cause cardiovascular morbidity by direct myocardial injury as a result of the inflammatory cascade or cytokine release, microvascular damage due to disseminated intravascular coagulation and thrombosis, direct entry of SARS-CoV-2 into myocardial cells via binding to ACE2 receptors, and hypoxemia combined with increased metabolic 
demands of acute illness leading to myocardial injury [911]. In this prospective cohort study we further assess the interaction of ECG abnormalities and Troponin elevation. We demonstrate that (1) myocardial injury defined by elevated Troponin is common among patients hospitalized with COVID-19 but is more often mild, associated with low-level elevation in troponin concentration. (2) more significant myocardial injury, as evident by increased Troponin level may be associated with higher risk of mortality. (3) In the group of patients with mild Troponin elevation $(0.05-1 \mathrm{ng} / \mathrm{ml})$, ECG abnormalities are associated with significantly increased mortality.

A variety of electrocardiographic manifestations have been documented in COVID-19. In patients with preexisting heart disease, the spectrum of ECG changes, from common atrial arrhythmias, precipitated by acute systemic illness to conduction system abnormalities and life-threatening arrhythmias in severe disease, besides other manifestations such as acute coronary syndrome, myocarditis and heart failure, are noted. ${ }^{24}$ In addition to the disease process, biochemical abnormalities like electrolyte imbalance, especially in critically ill patients and potential treatment modalities employed in COVID19 management, such as hydroxychloroquine and other antiviral drugs, may have varied impact on the electrocardiographic parameters such as the QT interval.

In our study, only $8.2 \%$ of the patients had prior Coronary artery disease, yet about $57.1 \%$ of the patients showed abnormal ECG at admission in the hospital indicating an acute COVID-19 disease process as the potential cause of the electrocardiographic changes. In a case report by Zhung et al ECG changes documented in a COVID-19 patient with fulminant myocarditis were sinus tachycardia and Right bundle branch block. RBBB pattern without Significant ST-T wave abnormalities. ${ }^{25} \mathrm{Kim}$ et al have reported nonspecific interventricular conduction delay (IVCD) and premature complexes in a 21-year female with COVID-19 related myocarditis while in few other patients, ST elevation seen were in inferior leads. ${ }^{26,27}$ Diffuse ST segment elevation in inferolateral leads and ST depression with T inversion in V1 and aVR were the ECG findings in a study done by Inciardi et al. ${ }^{6}$

Arrhythmias were reported more in the COVID-19 patients with severe disease than those with mild disease $(44.4 \%$ vs $6.9 \%$ p <0.001). ${ }^{22}$ While atrial premature complexes and ventricular premature complexes accounted for $3.1 \%$ and $4.1 \%$ of abnormal ECG changes in our study, conduction abnormalities such as Atrioventricular block (AV block), left bundle branch block (LBBB) and Right bundle branch block (RBBB) constituted only about $5 \%$. In our study, sinus tachycardia was seen in $55.2 \%(n=53)$ of the patients. Sinus tachycardia is noted to be the frequent manifestation in patients with COVID-19. Wang et al in their study comparing ECG changes in severe and critically ill COVID-19 patient found out sinus tachycardia to be the second most frequent ECG characteristic, more so in critically ill patients.

The relationship between ST-T changes on ECG and myocardial damage with poor prognosis has been demonstrated. ${ }^{34}$ Abnormal ST segment deviation (elevation/depression) and $\mathrm{T}$ inversion together, were the most common (26.9\%) abnormal ECG manifestation in our study. Also, these changes had statistically significant difference among severe and non-severe groups $(p=0.001)$. Patients with ST segment depression on admission ECG were more likely to progress to severe disease needing referral to State COVID ICU. Similar results were found out by Wang et al in their study of severe and critically severe patient groups of COVID-19, in which ST-T abnormal changes were the most common ECG manifestation. They also noticed that such changes were more pronounced in critically severe patients compared to severe patients. ${ }^{28}$ Similarly, in another study ST depression, T wave inversion were observed more in severe group than in non-severe group. ${ }^{35}$ ST-T changes may have various pathological basis such as myocardial damage inflicted by SARS CoV2, hypocalcaemia, hypertension, or coronary heart disease. Thus, recognition of such abnormal ECG manifestations may guide the treating physicians in early identification of impending severe COVID-19 among infected patients.

Though troponin elevation above the 99th percentile of the upper reference limit is considered the central marker of "myocardial injury", mild elevation between 0.05 to 1 is often nonspecific and associated with non-vascular etiologies such as strain, myocyte necrosis and increased cell membrane permeability [12]. Indeed, mild Troponin elevation was a frequent finding in our cohort, present in $31 \%$ of patients with COVID19. In this regard, our data suggests that assessment for the presence of ECG abnormalities can be used to enhance inpatient risk stratification in those patients with mild Troponin elevation.

This prospective study demonstrated that, more than half of the patients admitted with COVID-19 have elevated Troponin I level and abnormal ECG at admission. Both these parameters were significantly associated with poor in-hospital outcome in patients hospitalized with COVID 
19. This was in agreement with the study of Ghaleb et al. who observed that, more than half of the patients were admitted to intensive care unit; cTnl level was elevated in 30 patients $(58.8 \%)$, of whom 17 died $(56.7 \%)$. The study observed statistically significant differences regarding the relation between cTnl level and death. ${ }^{15}$ Deng et al. found the presence of myocardial injury in COVID-19 patients during hospitalization and one fourth of the patients, had presented abnormalities similar to myocarditis, with an increase in cardiac troponin I especially during hospitalization also in about $(37.5 \%)$ of them, especially in those who were died. Cardiac troponin levels were significantly increased one week before death. With normal findings in Echocardiography and ECG, they suggested that the increase in troponin was related to systemic disorders and could be the warning sign for the death of patients with COVID-19 and should be taken seriously in clinical practice. ${ }^{16}$ Recent data has shown that cTnl levels are mildly elevated in all patients with SARS-CoV-2 infection, but cTnl levels are markedly elevated in patients with severe SARS-CoV-2 infection compared to those with milder forms of the disease. ${ }^{17}$ Another study suggested that mild cTnl elevation was frequent in COVID-19 patients and in such cases presence or absence of ECG abnormalities coul be used to enhance risk stratification of hospitalized COVID patients. ${ }^{12}$

Limitations: The study had some limitations inherent to its design. As patients were selected purposively from selected hospital their might be a chance of selection bias. Baseline ECGs were not available in all patients, and it is possible that some ECG abnormalities were present prior to the diagnosis of COVID-19.

Conclusion: From this study it was concluded that cardiac troponin I and abnormal ECG have predictive potential for mortality in hospitalized COVID-19 patients. Myocardial injury is more commonly seen in COVID patients who died in the hospital in the form of elevated troponin levels and ECG abnormalities, and there is a positive correlation between death and elevated troponin levels and ECG abnormalities.

Recommendations: The study findings could be used to enhance risk stratification in patients hospitalized with COVID19. Close examination of cardiac status should be taken into account during managing such patients.

Conflict of interest: Nothing to declare.

\section{References:}

1. Wang D, Hu B, Hu C, Zhu F, Liu X, Zhang J, et al. Clinical Characteristics of 138 Hospitalized Patients with 2019 Novel Coronavirus-Infected Pneumonia in Wuhan, China. J Am Med Assoc. 2020; 323(11): 1061-9.

2. Xu Z, Shi L, Wang Y, Zhang J, Huang L, Zhang C, et al. Pathological findings of COVID-19 associated with acute respiratory distress syndrome. Lancet Respir Med. 2020;8(4):420-2.

3. Zheng Y-Y, Ma Y-T, Zhang J-Y, Xie X. COVID-19 and the cardiovascular system. Nat Rev Cardiol. 2020;17(5):259e260.

4. Hu B, Deng Q, Zhou Q. Cardiac involvement of COVID-19: Looking forward to novel discoveries and clinically valuable evidence. Int J Cardiol. 2020 Sep 1;314:95.

5. Channappanavar R, Perlman S. Pathogenic human coronavirus infections: causes and consequences of cytokine storm and immunopathology. Semin Immunopathol. 2017;39(5):529-539.

6. Efros O, Soffer S, LeibowitzA, Fardman A, Klempfner $R$, Meisel $E$, et al. Risk factors and mortality in patients with pneumonia and elevated troponin levels. Sci Rep. 2020; 10(1):21619.

7. Cangemi R, Calvieri C, Falcone M, Bucci T, Bertazzoni G, Scarpellini MG, et al. Relation of Cardiac Complications in the Early Phase of Community-Acquired Pneumonia to Long-Term Mortality and Cardiovascular Events. Am J Cardiol. 2015; 116(4):647-651.

8. Guo T, Fan Y, Chen M, Wu X, Zhang L, He T, et al. Cardiovascular implications of fatal outcomes of patients with coronavirus disease 2019 (COVID19). JAMA cardiology. 2020.

9. Lombardi CM, Carubelli V, Iorio A, Inciardi RM, Bellasi A, Canale C, et al. Association of Troponin Levels With Mortality in Italian Patients Hospitalized With Coronavirus Disease 2019: Results of a Multicenter Study. JAMA Cardiology. 2020.

10. Bhatla A, Mayer MM, Adusumalli S, Hyman MC, Oh E, Tierney A, et al. COVID-19 and cardiac arrhythmias. Heart Rhythm. 2020; 17(9):1439-44.

11. Liaqat, A., Ali-Khan, R.S., Asad, M. et al. Evaluation of myocardial injury patterns and ST changes among critical and non-critical patients with coronavirus-19 disease. Sci Rep.2021; 11: 4828.

12. Efros O, Barda N, Meisel E, LeibowitzA, Fardman A, Rahav G, et al. (2021) Myocardial injury in hospitalized patients with COVID-19 infectionRisk factors and outcomes. PLoS ONE 16(2): e0247800. 
13. Chorin E, Dai M, Kogan E, Wadhwani L, Shulman E, Nadeau-Routhier C, et al. Electrocardiographic Abnormalities and Troponin Elevation in COVID19.2021. medRxiv preprint doi: https://doi.org/ 10.1101/2020.11.12.20230565.

14. World Medical Association. Declaration of Helsinki: ethical principles for medical research involving human subjects. JAMA 310, 2191-2194 (2013).

15. DGHS. Coronavirus (COVID-19). Accessed: May 27, 2020. Available at https://www.dghs.gov.bd/ index.php/en/component/content/article?id=5393

16. Clerkin KJ, Fried JA, Raikhelkar J, et al. Coronavirus Disease 2019 (COVID-19) and Cardiovascular Disease. Circulation 2020. 18.

17. Patel AB, Verma A. COVID-19 and AngiotensinConverting Enzyme Inhibitors and Angiotensin Receptor Blockers: What Is the Evidence? JAMA 2020. 19.

18. Madjid M, Safavi-Naeini P, Solomon SD, Vardeny O. Potential Effects of Coronaviruses on the Cardiovascular System: A Review. JAMA Cardiology 2020.

19. Sia CH, Ngiam JN, Chew N, Beh DL, Poh KK. An educational case series of electrocardiographs during the COVID-19 pandemic and their implications on therapy. Singapore Med J. 2020;1:15.

20. Zeng JH, Liu YX, Yuan J, Wang FX, Wu WB, Li JX, et al. First case of COVID-19 complicated with fulminant myocarditis: a case report and insights. Infection. 2020:1.

21. Kim IC, Kim JY, Kim HA, Han S. COVID-19-related myocarditis in a 21-year-old female patient. Eur Heart J. 2020;41(19):1859.
22. Hu H, Ma F, Wei X, Fang Y. Coronavirus fulminant myocarditis treated with glucocorticoid and human immunoglobulin. Europ Heart J. 2020.

23. Inciardi RM, Lupi L, Zaccone G, Italia L, Raffo M, Tomasoni D, et al. Cardiac involvement in a patient with coronavirus disease 2019 (COVID-19). J Am Med Assoc Cardiol. 2020.

24. Liao Y, Llu K, Dyer A, Schoenberger JA, Shekelle R, Collette $P$, et al. Sex differential in the relationship of electrocardiographic ST-T abnormalities to risk of coronary death: 11.5 year follow-up findings of the Chicago heart association detection pro- ject in industry. Circulation. 1987;75(2):347-52.

25. Deng Q, Hu B, Zhang Y, Wang H, Zhou X, Hu W, et al. Suspected myocardial injury in patients with COVID-19: Evidence from front-line clinical observation in Wuhan, China. International journal of cardiology. 2020.

26. Korff S, Katus HA, Giannitsis E. Differential diagnosis of elevated troponins. Heart. 2006;92(7):987-993. doi:10.1136/hrt.2005.071282

27. Ghaleb AA, Sadky A, Meghaizel MA, El Etriby S. Prognostic Value of Troponin I in COVID-19 Patients - The Open Cardiovascular Medicine Journal. 2021;15:18-22.

28. Deng Q, Hu B, Zhang Y, et al. Suspected myocardial injury in patients with COVID-19: Evidence from front-line clinical observation in Wuhan, China. Int J Cardiol 2020; 311: 116-21.

29. Lippi G, Plebani M. Laboratory abnormalities in patients with COVID-2019 infection. Clin Chem Lab Med 2020; 58(7): 1131-4. 